\title{
Gaussian approximations for the exchange-energy functional of current-carrying states: Applications to two-dimensional systems
}

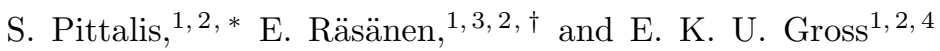 \\ ${ }^{1}$ Institut für Theoretische Physik, Freie Universität Berlin, Arnimallee 14, D-14195 Berlin, Germany \\ ${ }^{2}$ European Theoretical Spectroscopy Facility (ETSF) \\ ${ }^{3}$ Nanoscience Center, Department of Physics, University of Jyväskylä, FI-40014 Jyväskylä, Finland \\ ${ }^{4}$ Max-Planck-Institut für Mikrostrukturphysik, Weinberg 2, D-06120 Halle, Germany
}

(Dated: October 27, 2018)

\begin{abstract}
Electronic structure calculations are routinely carried out within the framework of densityfunctional theory, often with great success. For electrons in reduced dimensions, however, there is still a need for better approximations to the exchange-correlation energy functional. Furthermore, the need for properly describing current-carrying states represents an additional challenge for the development of approximate functionals. In order to make progress along these directions, we show that simple and efficient expressions for the exchange energy can be obtained by considering the short-range behavior of the one-body spin-density matrix. Applications to several two-dimensional systems confirm the excellent performance of the derived approximations, and verify the gaugeinvariance requirement to be of great importance for dealing with current-carrying states.
\end{abstract}

PACS numbers: 31.15.E-, 71.15.Mb

\section{INTRODUCTION}

The success of density-functional theory [1] (DFT) crucially depends on the availability of good approximations for the exchange-correlation (xc) energy functional. Great progress has been achieved beyond the commonly used local (spin) density approximation [L(S)DA] by means of, e.g., generalized-gradient approximations, orbital functionals, and hybrid functionals [2]. However, most of the approximations developed so far have focused on three-dimensional (3D) systems.

Most density functionals developed for 3D perform poorly when applied to two-dimensional (2D) systems [3, 4, 5, 6]. However, at present 2D structures constitute a large pool of applications in semiconductor nanotechnology, e.g., quantum Hall systems, spintronic devices, and quantum dots (QDs) 7]. Within DFT these systems are often treated using the 2D form of the LSDA employing the analytic expression of exchange energy of the 2D electron gas [8] (2DEG) and the corresponding correlation energies parametrized via quantum Monte Carlo calculations first by Tanatar and Ceperley [9] and later, for the complete range of collinear spin polarization, by Attaccalite et al [10]. Despite the relatively good performance of the LSDA in terms of total energies, there is still a clear need for accurate density functionals in 2D. Only recently, xc functionals tailored for 2D have been pushed forward [11, 12, 13, 14, 15, 16]. Here we make another natural step along these directions by studying Gaussian approximations (GAs) for the exchange energy functional. We extend previous studies on GAs [17, 18, 19, 20, 21, 22] to (i) 2D systems and to

*Electronic address: pittalis@physik.fu-berlin.de

${ }^{\dagger}$ Electronic address: erasanen@jyu.fi (ii) current-carrying states for both $2 \mathrm{D}$ and $3 \mathrm{D}$ systems. Numerical tests of the resulting exchange energies show significant improvement over the LSDA.

\section{GENERAL FORMALISM}

Within current-spin-density functional theory [27, 28] (CSDFT), the ground state energy $E_{v}$, the spin densities $\rho_{\sigma}(\mathbf{r})$ and paramagnetic current density $\mathbf{j}_{\sigma}(\mathbf{r})$ of a system of $N=N_{\uparrow}+N_{\downarrow}$ interacting electrons are determined by minimizing the total-energy functional which is given in Hartree atomic units as

$$
\begin{gathered}
E_{v}\left[\left\{\rho_{\sigma}, \mathbf{j}_{\sigma}\right\}\right]=T_{s}\left[\left\{\rho_{\sigma}, \mathbf{j}_{\sigma}\right\}\right]+E_{\mathrm{H}}[\rho] \\
+\sum_{\sigma=\uparrow, \downarrow} \int d^{D} r v_{\sigma}(\mathbf{r}) \rho_{\sigma}(\mathbf{r})+\frac{1}{c} \sum_{\sigma=\uparrow, \downarrow} \int d^{D} r \mathbf{A}_{\sigma}(\mathbf{r}) \mathbf{j}_{\sigma}(\mathbf{r}) \\
+\frac{1}{2 c} \sum_{\sigma=\uparrow, \downarrow} \int d^{D} r \mathbf{A}_{\sigma}^{2}(\mathbf{r}) \rho_{\sigma}(\mathbf{r})+E_{x c}\left[\left\{\rho_{\sigma}\right\},\left\{\mathbf{j}_{\sigma}\right\}\right]
\end{gathered}
$$

where $D=2,3$ is the dimensionality of interest, $T_{s}\left[\left\{\rho_{\sigma}, \mathbf{j}_{\sigma}\right\}\right]$ is the kinetic energy functional of noninteracting particles, $v_{\sigma}(\mathbf{r})$ is an external (local) spindependent potential acting upon the interacting system, $\mathbf{A}_{\sigma}(\mathbf{r})$ is an external (local) spin-dependent vector potential (note that physical fields are spin-independent, but it is formally convenient to introduce the spin-dependence), $E_{\mathrm{H}}[\rho]$ is the classical electrostatic or Hartree energy of the total charge density $\rho(\mathbf{r})=\rho_{\uparrow}(\mathbf{r})+\rho_{\downarrow}(\mathbf{r})$, and $E_{x c}\left[\left\{\rho_{\sigma}, \mathbf{j}_{\sigma}\right\}\right]$ is the xc energy functional. This quantity is usually further decomposed into the exchange and correlation energies as

$$
E_{x c}\left[\left\{\rho_{\sigma}, \mathbf{j}_{\sigma}\right\}\right]=E_{x}\left[\left\{\rho_{\sigma}, \mathbf{j}_{\sigma}\right\}\right]+E_{c}\left[\left\{\rho_{\sigma}, \mathbf{j}_{\sigma}\right\}\right]
$$


An exact expression is known for the exchange energy

$$
E_{x}\left[\left\{\rho_{\sigma}, \mathbf{j}_{\sigma}\right\}\right]=-\frac{1}{2} \sum_{\sigma=\uparrow, \downarrow} \int d^{D} r_{1} \int d^{D} r_{2} \frac{\left|\gamma_{\sigma}\left(\mathbf{r}_{1}, \mathbf{r}_{2}\right)\right|^{2}}{\left|\mathbf{r}_{1}-\mathbf{r}_{2}\right|}
$$

where $\gamma_{\sigma}$ is the one-body spin-density matrix (1BSDM) of the Kohn-Sham (KS) system

$$
\gamma_{\sigma}\left(\mathbf{r}_{1}, \mathbf{r}_{2}\right)=\sum_{k=1}^{N_{\sigma}} \psi_{k, \sigma}\left(\mathbf{r}_{1}\right) \psi_{k, \sigma}^{*}\left(\mathbf{r}_{2}\right)
$$

and $\psi_{k \sigma}(\mathbf{r})$ are the KS spin orbitals. Note that we are assuming that the KS ground state has the form of a single Slater determinant. The number of electrons with spin $\sigma$ is given by

$$
N_{\sigma}=\int d^{D} r_{1} \int d^{D} r^{\prime}\left|\gamma_{\sigma}\left(\mathbf{r}_{1}, \mathbf{r}^{\prime}\right)\right|^{2}
$$

Introducing the average and relative particle coordinates, respectively, as

$$
\mathbf{r}=\frac{1}{2}\left(\mathbf{r}_{1}+\mathbf{r}_{2}\right), \mathbf{s}=\mathbf{r}_{1}-\mathbf{r}_{2}
$$

Eq. (3) can be rewritten as

$$
E_{x}\left[\rho_{\uparrow}, \rho_{\downarrow}\right]=-\frac{\Omega_{D}}{2} \sum_{\sigma=\uparrow, \downarrow} \int d^{D} r \int s^{(D-2)} d s\left\langle\left|\gamma_{\sigma}\right|^{2}\right\rangle,
$$

where

$$
\left\langle\left|\gamma_{\sigma}\right|^{2}\right\rangle=\frac{1}{\Omega_{D}} \int d \Omega_{D}\left|\gamma_{\sigma}\left(\mathbf{r}+\frac{\mathbf{s}}{2}, \mathbf{r}-\frac{\mathbf{s}}{2}\right)\right|^{2}
$$

is the angular average of $\left|\gamma_{\sigma}\right|^{2}$. Note that $\left\langle\left|\gamma_{\sigma}\right|^{2}\right\rangle$ is a function of the position, $\mathbf{r}$, but this fact is omitted in the notation. Also in the remaining part of the present work, we often shorten the notation where the dependence on $\mathbf{r}$ is clear. The term $d \Omega_{D}$ in Eq. (8) indicates the angular differential in $D$ dimensions, $\Omega_{2}=2 \pi$, and $\Omega_{3}=4 \pi$. In the new coordinates, Eq. (5) can be rewritten as

$$
N_{\sigma}=\Omega_{D} \int d^{D} r \int s^{(D-1)} d s\left\langle\left|\gamma_{\sigma}\right|^{2}\right\rangle .
$$

It is apparent that an approximation for $\left\langle\left|\gamma_{\sigma}\right|^{2}\right\rangle$ in Eq. (8) provides an approximation for the exchange energy in Eq. (7). Equation (9) allows us to check the validity of such an approximation in terms of the particle-number normalization.

\section{GAUSSIAN APPROXIMATION FOR THE ONE-BODY SPIN-DENSITY MATRIX}

One of the more successful strategies to develop approximation for the exchange energy functional is based on the density-matrix expansion scheme. [23, 24, 25, 26]
Here we reconsider the so-called short-range behavior of the 1BSDM. In particular, we extend the results previously derived for zero-current states in $3 \mathrm{D}$ [18, 19] to current-carrying states of both $3 \mathrm{D}$ and $2 \mathrm{D}$ systems.

In dealing with current-carrying states it is necessary to allow the orbitals in Eq. (4) to be complex valued. Expanding the 1BSDM in the inter-particle coordinate, $\mathbf{s}$, at a given average position, $\mathbf{r}$, we find

$$
\gamma_{\sigma}\left(\mathbf{r}+\frac{\mathbf{s}}{2}, \mathbf{r}-\frac{\mathbf{s}}{2}\right) \approx \rho_{\sigma}(\mathbf{r})\left\{1-\frac{\mathbf{s}^{T} \mathbf{\Sigma}_{\sigma} \mathbf{s}}{\rho_{\sigma}}+i \frac{\mathbf{j}_{\sigma}^{T} \mathbf{s}}{\rho_{\sigma}}\right\}
$$

where

$$
\mathbf{j}_{\sigma}=\frac{1}{2 i} \sum_{k=1}^{N_{\sigma}}\left[\psi_{k \sigma}^{*}\left(\nabla \psi_{k \sigma}\right)-\left(\nabla \psi_{k \sigma}^{*}\right) \psi_{k \sigma}\right]
$$

is the spin-dependent (paramagnetic) current density, and

$$
\left[\boldsymbol{\Sigma}_{\sigma}\right]_{\alpha, \beta}=\left[t_{\sigma}\right]_{\alpha, \beta}+\frac{1}{2} \sum_{k=1}^{N_{\sigma}} \frac{j_{k \sigma, \alpha} j_{k \sigma, \beta}}{\rho_{k \sigma}}
$$

with

$$
\left[t_{\sigma}\right]_{\alpha, \beta}=\frac{1}{8}\left[\sum_{k=1}^{N_{\sigma}} \frac{1}{\rho_{k \sigma}} \frac{\partial \rho_{k \sigma}}{\partial x_{\alpha}} \frac{\partial \rho_{k \sigma}}{\partial x_{\beta}}-\frac{\partial^{2}}{\partial x_{\alpha} \partial x_{\beta}} \rho_{\sigma}\right],
$$

where $\alpha, \beta$ runs over the Cartesian coordinates in 3D or $2 \mathrm{D}$. The term $\left[\boldsymbol{\Sigma}_{\sigma}\right]_{\alpha, \beta}$ can be interpreted as kinetic energy-density tensor. We point out that $\boldsymbol{\Sigma}_{\sigma}$ is not a gauge-invariant quantity due to the second term in Eq. (12).

At this point, we carry out an exponential resummation of Eq. (10), which yields

$$
\tilde{\gamma}_{\sigma}=\rho_{\sigma} \exp \left[-\frac{\mathbf{s}^{T} \boldsymbol{\Sigma}_{\sigma} \mathbf{s}}{\rho_{\sigma}}+i \frac{\mathbf{j}_{\sigma}^{T} \mathbf{s}}{\rho_{\sigma}}\right]
$$

where $\mathbf{s}^{T}$ and $\mathbf{j}^{T}$ are the transposes of the vectors $\mathbf{s}$ and $\mathbf{j}$, respectively. Although this approximation reproduces the exact short range behavior of the 1BSDM, it seems at first sight - a rather oversimplified approximation for arbitrary s. However, it has the appealing feature that the corresponding Wigner transform [1] reproduces the spin-particle density, the paramagnetic current density, and the kinetic-energy-density tensor of the KS system exactly (see below).

The Wigner transformation of Eq. (14) is given by

$$
\begin{aligned}
\tilde{f}_{\sigma}^{W}(\mathbf{r}, \mathbf{p}) & \approx \frac{\rho_{\sigma}}{\sqrt{(2 \pi)^{D} \operatorname{det}\left(k_{B} \boldsymbol{\Theta}_{\sigma}\right)}} \\
& \times \exp \left[-\frac{1}{2}\left(\mathbf{p}-\frac{\mathbf{j}_{\sigma}}{\rho_{\sigma}}\right)^{T}\left(k_{B} \boldsymbol{\Theta}_{\sigma}\right)^{-1}\left(\mathbf{p}-\frac{\mathbf{j}_{\sigma}}{\rho_{\sigma}}\right)\right](15)
\end{aligned}
$$

where, following Ref. [19], we define the matrix $\boldsymbol{\Theta}_{\sigma}$ to satisfy the relation

$$
\frac{1}{2} k_{B} \rho_{\sigma} \boldsymbol{\Theta}_{\sigma}=\boldsymbol{\Sigma}_{\sigma},
$$


where $k_{B}$ is the Boltzmann constant. The presence of the Boltzmann constant is motivated by the fact that for the spin-unpolarized zero-current case, the Wigner transform in question provides a description for the KS state formally resembling a thermodynamic one $17,18,19,20$, 21, 22]. Instead, in this work we allow the orbitals to be spin-unrestricted and complex valued. We observer that $\boldsymbol{\Theta}_{\sigma}$ is apparently not gauge-invariant, thus we shall refrain from interpreting it as a local temperature. Finally, it is immediate to verify that

$$
\begin{gathered}
\rho_{\sigma}(\mathbf{r})=\int d^{D} p \tilde{f}_{\sigma}^{W}(\mathbf{r}, \mathbf{p}), \\
\mathbf{j}_{\sigma}(\mathbf{r})=\int d^{D} p \mathbf{p} \tilde{f}_{\sigma}^{W}(\mathbf{r}, \mathbf{p}),
\end{gathered}
$$

and

$$
\begin{aligned}
{\left[\boldsymbol{\Sigma}_{\sigma}\right]_{\alpha, \beta}(\mathbf{r}) } & =\frac{1}{2} \int d^{D} p\left(p_{\alpha}-\frac{j_{\sigma, \alpha}(\mathbf{r})}{\rho_{\sigma}(\mathbf{r})}\right)\left(p_{\beta}-\frac{j_{\sigma, \beta}(\mathbf{r})}{\rho_{\sigma}(\mathbf{r})}\right) \\
& \times \tilde{f}_{\sigma}^{W}(\mathbf{r}, \mathbf{p}) .
\end{aligned}
$$

\section{GAUSSIAN APPROXIMATIONS FOR THE EXCHANGE ENERGIES}

In this section, we provide few approximations for the exchange energy of spin-polarized current-carrying states. Although what follows may be applied to the 3D case as well, we shall restrict ourselves to 2D systems where the need of new approximations in the quantumHall regime, for example, is particularly large.

Let us first observe that substitution of Eq. (14) in Eq. (8) would yield an expression which is not gauge invariant. Popular (3D) meta-generalized gradient approximations for the xc energy are also not gauge invariant 29]. This fact is not only formally inadequate [27, 28], but it is also a source of practical problems. For example, it has been observed that the lack of gauge invariance causes a wrong description of degenerate atomic ground states carrying different paramagnetic currents. Recently, Tao and Perdew have proposed a correction for this problem [29, 30]. Here we show how this kind of correction naturally emerges in the context of the present GA.

In order to fulfill the gauge invariance requirement, we substitute Eq. (10) in Eq. (8), and obtain

$$
\left\langle\left|\gamma_{\sigma}\right|^{2}\right\rangle \approx \rho_{\sigma}^{2}\left\{1-\left[\frac{\operatorname{Tr}\left(\boldsymbol{\Sigma}_{\sigma}\right)}{\rho_{\sigma}}-\frac{1}{2}\left(\frac{\mathbf{j}_{\sigma}}{\rho_{\sigma}}\right)^{2}\right] s^{2}\right\}
$$

where

$$
\begin{aligned}
\operatorname{Tr}\left(\boldsymbol{\Sigma}_{\sigma}\right) & =\sum_{k=1}^{N_{\sigma}}\left[\frac{1}{8} \frac{\left(\nabla \rho_{k \sigma}\right)^{2}}{\rho_{k \sigma}}+\frac{1}{2} \frac{\mathbf{j}_{k \sigma}^{2}}{\rho_{k \sigma}}\right]-\frac{1}{8} \nabla^{2} \rho_{\sigma} \\
& =\tau_{\sigma}-\frac{1}{8} \nabla^{2} \rho_{\sigma},
\end{aligned}
$$

with

$$
\tau_{\sigma}=\frac{1}{2} \sum_{k=1}^{N_{\sigma}}\left|\nabla \psi_{k, \sigma}\right|^{2}
$$

being the spin-dependent kinetic-energy density. Note that, while $\tau_{\sigma}$ is not gauge invariant, the modified quantity

$$
\tilde{\tau}_{\sigma}=\tau_{\sigma}-\frac{1}{2}\left(\frac{\mathbf{j}_{\sigma}^{2}}{\rho_{\sigma}}\right)
$$

is indeed gauge invariant. Now, let us perform an exponential resummation of Eq. (20), which leads to the following GA

$$
\left\langle\left|\gamma_{\sigma}\right|^{2}\right\rangle \approx \rho_{\sigma}^{2} \exp \left(-\frac{s^{2}}{\beta_{\sigma}}\right)
$$

where

$$
\beta_{\sigma}^{-1}=\left[\frac{\tau_{\sigma}}{\rho_{\sigma}}-\frac{1}{8} \frac{\nabla^{2} \rho_{\sigma}}{\rho_{\sigma}}-\frac{1}{2}\left(\frac{\mathbf{j}_{\sigma}}{\rho_{\sigma}}\right)^{2}\right],
$$

or, alternatively, using Eq. (16)

$$
\beta_{\sigma}^{-1}=\frac{1}{2}\left[\operatorname{Tr}\left(\Theta_{\sigma}\right)-\left(\frac{\mathbf{j}_{\sigma}}{\rho_{\sigma}}\right)^{2}\right] .
$$

Expression (26) shows how $\beta_{\sigma}$ relates to $\boldsymbol{\Theta}_{\sigma}$. In contrast to $\boldsymbol{\Theta}_{\sigma}, \beta_{\sigma}$ is a gauge-invariant quantity. Hence, the approximation for the exchange energy obtained from Eq. (26) is gauge invariant as well.

We further focus on inhomogeneous 2D systems. An expression for the exchange energy is readily obtained by inserting Eq. (24) in Eqs. (7) and (91):

$$
E_{x}\left[\rho_{\uparrow}, \rho_{\downarrow}\right]=-\frac{\pi^{3 / 2}}{2} \sum_{\sigma=\uparrow, \downarrow} \int d^{2} r \rho_{\sigma}^{2}(\mathbf{r}) \beta_{\sigma}^{1 / 2}(\mathbf{r}) .
$$

This is a functional for the exchange energy in the form of a current-dependent meta-generalized gradient approximation. To stress the dependency on the paramagnetic current, and to remind its origin from a GA, we will indicate the functional as J-GA.

Furthermore, we impose that our approximation reproduces the correct normalization condition (5). Hence, we modify Eq. (24) by adding a fourth-order term [20] as follows

$$
\left\langle\left|\gamma_{\sigma}\right|^{2}\right\rangle \approx \rho_{\sigma}^{2} \exp \left(-\frac{s^{2}}{\beta_{\sigma}}\right)\left[1+A_{\sigma}\left(\frac{s}{\beta_{\sigma}}\right)^{2}\right] .
$$

This expression leads to

$$
\begin{aligned}
E_{x}\left[\rho_{\uparrow}, \rho_{\downarrow}\right] & =-\frac{\sqrt{\pi}}{2} \sum_{\sigma=\uparrow, \downarrow} \int d^{2} r\left[\pi+\frac{3}{4} \sqrt{\pi} A_{\sigma}\right] \\
& \times \rho_{\sigma}^{2}(\mathbf{r}) \beta_{\sigma}^{1 / 2}(\mathbf{r}) .
\end{aligned}
$$


The parameter $A_{\sigma}$ is determined by using the following relation

$$
N_{\sigma}=\pi \int d^{2} r\left[1+2 A_{\sigma}\right] \rho_{\sigma}^{2}(\mathbf{r}) \beta_{\sigma}(\mathbf{r}) .
$$

Eq. (29) together with Eq. (30) provide another density functional for the exchange energy. We refer to this functional as the current-dependent modified GA denoted by J-MGA.

\section{APPLICATIONS}

In order to test the performance and the degree of universality of the J-GA and J-MGA, given in Eqs. (27) and (29), respectively, we consider below different 2D electron systems, i.e., QDs of different shapes, as well as the 2DEG. We also assess the importance of the approximation to account for the paramagnetic current density in Eqs. (20) and (21), $\mathbf{j}_{\sigma}$, and thus the prerequisite of the gauge invariance. The assessment is done by comparing J-GA and J-MGA to the results obtained by neglecting in the functionals the terms depending explicitly on $\mathbf{j}_{\sigma}$. These approximations are denoted below as 0-GA and 0 -MGA.

\section{A. Finite systems}

As relevant examples of finite $2 \mathrm{D}$ electron systems we consider parabolic (harmonic) [7] and rectangular QDs [31, 32], respectively. In both cases, the manyelectron Hamiltonian is given by

$$
H=\sum_{i=1}^{N}\left[\frac{1}{2}\left(\mathbf{p}_{i}+\frac{1}{c} \mathbf{A}_{i}\right)^{2}+V_{\text {ext }}\left(\mathbf{r}_{i}\right)\right]+\sum_{i<j}^{N} \frac{1}{\left|\mathbf{r}_{i}-\mathbf{r}_{j}\right|}
$$

where $N$ is the number of electrons and $\mathbf{A}$ is the external vector potential of the homogeneous magnetic field $\mathbf{B}=$ $B \hat{z}$ perpendicular to the $2 \mathrm{D}(x-y)$ plane.

The external confining potential is defined for a parabolic QD, containing here $N=2 \ldots 20$ electrons, as

$$
V_{\mathrm{ext}}^{\mathrm{par}}(r)=\frac{1}{2} \omega_{0}^{2} r^{2}
$$

where $\omega_{0}$ is the confinement strength. For $N=2$ we have $\omega_{0}=1$ (see below), and otherwise $\omega_{0}=0.42168$ corresponding to a typical confinement of $5 \mathrm{meV}$ when applying the effective mass approximation (with the effective mass $m^{*}=0.067 m_{e}$ and dielectric constant $\left.\epsilon=12.4 \epsilon_{0}\right)$ in the modeling of GaAs QDs [7].

The rectangular $\mathrm{QD}$ containing $N=4 \ldots 16$ electrons is defined by

$$
V_{\text {ext }}^{\text {rec }}(x, y)=\left\{\begin{array}{l}
0, \quad 0 \leq x \leq \alpha L, 0 \leq y \leq L \\
\infty, \quad \text { elsewhere }
\end{array}\right.
$$

TABLE I: Exchange energies for closed-shell parabolic (upper part) and rectangular (lower part) quantum dots calculated using the exact exchange (EXX) (in the KLI approximation for $N>2$ ), the Gaussian approximation (GA), the modified Gaussian approximation (MGA), and the local spin-density approximation (LSDA). The relative errors with respect to EXX are also shown.

\begin{tabular}{cc|cc|cc|cc}
\hline \hline$N$ & EXX & J-GA & $\Delta(\%)$ & J-MGA & $\Delta(\%)$ & LSDA & $\Delta(\%)$ \\
\hline 2 & -1.08 & -1.12 & -3.0 & -1.10 & -1.5 & -0.98 & 9.3 \\
6 & -2.23 & -2.28 & -2.1 & -2.28 & -2.2 & -2.13 & 4.4 \\
12 & -4.89 & -5.01 & -2.5 & -5.03 & -2.9 & -4.76 & 2.6 \\
20 & -8.78 & -9.00 & -2.5 & -9.05 & -3.0 & -8.63 & 1.7 \\
\hline 6 & -3.14 & -3.33 & -5.9 & -3.25 & -3.3 & 2.99 & 4.9 \\
12 & -8.19 & -8.46 & -3.3 & -8.42 & -2.8 & -7.99 & 2.5 \\
16 & -12.7 & -13.3 & -4.4 & -13.1 & -3.2 & -12.3 & 3.5 \\
\hline
\end{tabular}

where $L=\sqrt{2} \pi$ is the (smaller) side length, and $\alpha=2$ determines the side ratio of the rectangle. The size is then $\sim 90 \mathrm{~nm} \times 45 \mathrm{~nm}$. The electronic properties of similar rectangular QDs have been studied in detail in Refs. 31] and [32].

We point out that, in principle, the presence of $\mathbf{A}$ in the Hamiltonian brings out the need of the full-flagged current-spin-density functional theory [27]. However, at the level of the KS equations we neglect the xc vector potential, but still include the external one. This approximation has been shown to be accurate for atomistic systems, and QDs up to relatively high magnetic fields [11, 12, 33, 34, 35, 36, 37, 38].

As reference we use the exact-exchange (EXX) results calculated in the Krieger-Li-Iafrate (KLI) approximation [39]. The converged KS orbitals from the KLI are then used as the input for our functionals. An exception is the two-electron parabolic QD with $\omega_{0}=1$, for which we can apply the known analytic density [40] in the calculation of the EXX energy (which is minus half of the Hartree energy) and as the input in the functionals. For comparison, we also compute the exchange energies from the (2D) LSDA [8]. Both the EXX and LSDA calculations are performed using the octopus real-space code within SDFT [41].

Table I shows the exchange energies for a set of both parabolic (upper part) and rectangular (lower part) QDs. The relative errors with respect to EXX are also shown. All the cases correspond to closed-shell ground-state solutions possessing no currents, and the total spin is $S=0$. Therefore, the J-GA (J-MGA) result is similar to 0-GA (0-MGA). Overall, we find a good agreement between the J-GA and the EXX. The J-MGA, which contains the fourth-order term in $\left\langle\left|\gamma_{\sigma}\right|^{2}\right\rangle$, is in most examples more accurate: The deviation from the EXX is $\lesssim 3 \%$. As expected, the LSDA generally overestimates the exchange energies, but the deviation from the EXX reduces as a function of $N$. In contrast, J-(M)GA seems to gradually lose its accuracy when $N$ is increased, especially in parabolic QDs. This may be due to the fact that since the electron density profile is relatively flat in large QDs, 
the LSDA correspondingly becomes more suitable.

In Table [I] we show the exchange energies for spinpolarized current-carrying states in parabolic (upper part) and rectangular (lower part) QDs. The $N=2$ QD with $S=1$ corresponds to an excited state, whereas in the other systems the spin polarization of the ground state is achieved by applying the external magnetic field. Compared to the EXX, the accuracy of both J-GA and J-MGA is excellent. Moreover, both functionals are considerably more accurate than the LSDA. On the other hand, the approximations 0-GA and 0-MGA obtained by omitting the current-dependent terms in Eq. (20) lead to completely wrong results. This demonstrates the importance of the currents involved in the derivation of a gauge invariant GA.

\section{B. Two-dimensional electron gas}

Finally let us consider the GA for the exchange energy in the case of the 2DEG. We restrict ourselves to the case of vanishing external vector potential, but still we allow the external scalar potential to be spin-dependent. In this situation, we can set $\nabla^{2} \rho_{\sigma}=0$ and $\nabla \rho_{\sigma}=0$. Moreover, we may set $\mathbf{j}_{\sigma}=0$ by considering the $2 \mathrm{DEG}$ at rest. Hence, the current term in Eq. (25) drops out from the final expression

$$
\left\langle\left|\gamma_{\sigma}\right|^{2}\right\rangle \approx \rho_{\sigma}^{2} \exp \left(-\pi \rho_{\sigma}^{2} s^{2}\right)
$$

We note that correct particle numbers for each spin channel are obtained from Eq. (34), and therefore $A_{\sigma}=0$. Defining the 2D density parameter $r_{s}=1 / \sqrt{\pi \rho}$, and the polarization $\xi=\left(\rho_{\uparrow}-\rho_{\downarrow}\right) / \rho$, the total exchange energy per particle becomes

$$
\epsilon_{x}\left[r_{s}, \xi\right]=-\frac{\sqrt{\pi}}{4 \sqrt{2} r_{s}}\left[(1+\xi)^{3 / 2}+(1-\xi)^{3 / 2}\right] .
$$

This expression can be used as an explicit density functional in the LSDA fashion. In fact, the only difference to the LSDA exchange [8] is the prefactor, which is here $\sim 4.4 \%$ smaller than in the LSDA. Therefore, one can immediately deduce from Tables \and I that, when considering finite QD systems of few electrons, Eq. (35) leads to good agreement with the EXX results by correcting the LSDA exchange energies by $\sim 4.4 \%$.

Interestingly, Eq. (35) has been recently obtained in an alternative way by considering the short-range behavior of the exchange-hole function [11]. This identity of the expression can be seen as a rather reassuring result.

\section{CONCLUSIONS}

In this work we have analyzed the one-body spindensity matrix for current-carrying states. We devised gauge-invariant approximations for the exchange energy by considering a Gaussian approximation for the short-range behavior of the one-body spin-density matrix, in particular, for its angular average of the module square. The resulting simple approximations for the exchange energy perform extremely well in a variety of twodimensional systems, including a diverse set of quantum dots, as well as the homogeneous two-dimensional electron gas. Moreover, our analysis confirms the relevance of the gauge invariance when dealing with current-carrying states.

\section{Acknowledgments}

This work was supported by the Deutsche Forschungsgemeinschaft, the EU's Sixth Framework Programme through the ETSF e-I3, and the Academy of Finland.
[1] For a review, see, e.g., R. G. Parr and W. Yang, Densityfunctional Theory of Atoms and Molecules (Oxford University Press - New York, Clarendon Press, Oxford, 1989); R. M. Dreizler and E. K. U. Gross, Density functional theory (Springer, Berlin, 1990).

[2] J. P. Perdew and S. Kurth, in A Primer in Density Functional Theory, edited by C. Fiolhais, F. Nogueira, and M. A. L. Marques (Springer, Berlin, 2003), p. 1; G. E. Scuseria and V. N. Staroverov, in: Theory and Applications of Computational Chemistry: The First Forty Years, edited by C. E. Dykstra, G. Frenking, K. S. Kim, and G. E. Scuseria (Elsevier, Amsterdam, 2005), p. 669.

[3] Y.-H. Kim, I.-H. Lee, S. Nagaraja, J.-P. Leburton, R. Q. Hood, and R. M. Martin, Phys. Rev. B 61, 5202 (2000).

[4] L. Pollack and J. P. Perdew, J. Phys.: Condens. Matter 12, 1239 (2000).

[5] L. A. Constantin, Phys. Rev. B 78, 155106 (2008).

[6] L. A. Constantin, J. P. Perdew, and J. M. Pitarke, Phys. Rev. Lett. 101, 016406 (2008); erratum 101, 269902(E)
(2008).

[7] For a review, see, e.g., L. P. Kouwenhoven, D. G. Austing, and S. Tarucha, Rep. Prog. Phys. 64, 701 (2001); S. M. Reimann and M. Manninen, Rev. Mod. Phys. 74, 1283 (2002).

[8] A. K. Rajagopal and J. C. Kimball, Phys. Rev. B 15, 2819 (1977).

[9] B. Tanatar, D. M. Ceperley, Phys. Rev. B 39, 5005 (1989).

[10] C. Attaccalite, S. Moroni, P. Gori-Giorgi, and G. B. Bachelet, Phys. Rev. Lett. 88, 256601 (2002).

[11] S. Pittalis, E. Räsänen, N. Helbig, and E. K. U. Gross, Phys. Rev. B 76, 235314 (2007).

[12] N. Helbig, S. Kurth, S. Pittalis, E. Räsänen, and E. K. U. Gross, Phys. Rev. B 77, 245106 (2008).

[13] S. Pittalis, E. Räsänen, and M. A. L. Marques, Phys. Rev. B 78, 195322 (2008).

[14] S. Pittalis, E. Räsänen, J. G. Vilhena, M. A. L. Marques, Phys. Rev. A 79, 012503 (2009). 
TABLE II: Exchange energies (in effective atomic units, see Sec. VA for fully spin-polarized $(S=N / 2)$, current-carrying states in parabolic (upper part) and rectangular (lower part) quantum dots. The results have been calculated using the exact exchange (EXX) in the KLI approximation, the current-dependent Gaussian approximation (J-GA), the current-dependent modified GA (J-MGA), the non-current-dependent GA (0-GA) and MGA (0-MGA), and the local spin-density approximation (LSDA). The relative errors with respect to EXX are also shown.

\begin{tabular}{ccc|cc|ccc|cc|cc|cc}
\hline \hline$N$ & $B(\mathrm{~T})$ & EXX & J-GA & $\Delta(\%)$ & J-MGA & $\Delta(\%)$ & 0-GA & $\Delta(\%)$ & $0-$ MGA & $\Delta(\%)$ & LSDA & $\Delta(\%)$ \\
\hline 2 & 0 & -1.30 & -1.26 & 3.0 & -1.29 & 1.2 & -1.17 & 9.8 & -1.33 & -2.3 & -1.21 & 6.8 \\
4 & 4 & -1.92 & -1.89 & 1.8 & -1.92 & 0.21 & -1.49 & 23 & -2.18 & -13 & -1.83 & 4.8 \\
6 & 6 & -3.26 & -3.21 & 1.5 & -3.26 & 0 & -2.26 & 31 & -3.99 & -22 & -3.11 & 4.5 \\
\hline 4 & 8 & -2.46 & -2.43 & 1.3 & -2.46 & 0 & -1.66 & 32 & -2.07 & 16 & -2.36 & 4.3 \\
6 & 10 & -4.30 & -4.26 & 1.0 & -4.31 & -0.19 & -2.71 & 37 & -3.51 & 18 & -4.14 & 3.9 \\
8 & 10 & -6.42 & -6.42 & 0 & -6.46 & -0.67 & -4.00 & 38 & -5.24 & 18 & -6.18 & 3.8 \\
12 & 12 & -11.6 & -11.6 & 0 & -11.7 & -0.86 & -6.67 & 42 & -9.30 & 20 & -11.1 & 3.6 \\
\hline
\end{tabular}

[15] E. Räsänen, S. Pittalis, C. R. Proetto, and E. K. U. Gross, Phys. Rev. B 79, 121305(R) (2009).

[16] S. Pittalis, E. Räsänen, C. R. Proetto, and E. K. U. Gross, Phys. Rev. B 79, 085316 (2009).

[17] S. K. Ghosh, M. Berkowitz, and R. G. Parr, Proc. Natl. Acad. Sci. USA 81, 8028 (1984).

[18] G. Kemister, Phys. Rev, A 34, 4480 (1986).

[19] M. Berkowitz, Chem. Phys. Lett. 129, 486 (1986).

[20] C. Lee, and R. G. Parr, Phys. Rev. A 35, 2377 (1987).

[21] R. G. Parr, K. Rupnik, and S. K. Ghosh, Phys. Rev. Lett. 56, 1555 (1986).

[22] P. W. Ayers, R.G. Parr, A. Nagy, Int. J. Quant. Chem. 90, 309 (2001).

[23] J. W. Negele and D. Vautherin, Phys. Rev. C 5, 1472 (1972).

[24] R. M. Koehl, G. K. Odom, and G. E. Scuseria, Mol. Phys. 87, 835 (1996).

[25] T. V. Voorhis and G. E. Scuseria, Mol. Phys. 92, 601 (1997).

[26] T. Tsuneda and K. Hirao, Phys. Rev. B 62, 15527 (2000).

[27] G. Vignale and M. Rasolt, Phys. Rev. Lett. 59, 2360 (1987).

[28] G. Vignale and M. Rasolt, Phys. Rev. B 37, 10685 (1988).

[29] J. Tao and J. P. Perdew, Phys. Rev. Lett. 95, 196403 (2005).

[30] J. Tao, Phys. Rev. B 71, 205107 (2005).

[31] E. Räsänen, H. Saarikoski, V. N. Stavrou, A. Harju, M.
J. Puska, and R. M. Nieminen, Phys. Rev. B 67, 235307 (2003).

[32] E. Räsänen, A. Harju, M. J. Puska, and R. M. Nieminen, Phys. Rev. B 69, 165309 (2004).

[33] S. Sharma, S. Pittalis, S. Kurth, S. Shallcross, J. K. Dewhurst, and E. K. U. Gross, Phys. Rev. B 76, 100401(R) (2007).

[34] S. Pittalis, S. Kurth, N. Helbig and E. K. U. Gross, Phys. Rev. A 74, 062511 (2006).

[35] A. M. Lee, N. C. Handy, and S. M. Colwell, J. Chem. Phys. 103, 10095 (1995).

[36] H. Saarikoski, E. Räsänen, S. Siljamäki, A. Harju, M. J. Puska, and R. M. Nieminen, Phys. Rev. B 67, 205327 (2003).

[37] K. Capelle, Phys. Rev. A 60, R733 (1999).

[38] E. Orestes, A. B. F. da Silva, K. Capelle, Int. J. Quantum Chem. 103, 516 (2005).

[39] J. B. Krieger, Y. Li, and G. J. Iafrate, Phys. Rev. A 46 , 5453 (1992).

[40] M. Taut, J. Phys. A 27, 1045 (1994).

[41] M. A. L. Marques, A. Castro, G. F. Bertsch, A. Rubio, Comput. Phys. Commun. 151, 60 (2003); A. Castro, H. Appel, M. Oliveira, C. A. Rozzi, X. Andrade, F. Lorenzen, M. A. L. Marques, E. K. U. Gross, and A. Rubio, Phys. Stat. Sol. (b) 243, 2465 (2006). 